\title{
A MULTI-MERGING GALAXY MRK 273 WITH HOT EXTENDED GASEOUS HALO AND AN EXTENDED SOFT X-RAY COMPANION
}

\author{
X.Y. XIA \\ Dept. of Physics, Tianjin Normal University, P.R.China \\ Z.G. DENG AND H. WU \\ Beijing Astronomical Observatory, P.R. China \\ AND \\ T. BOLLER \\ Max-Planck-Institut für Extraterrestrische Physik,Germany
}

The ultraluminous IRAS galaxy Mrk 273 is a Seyfert 2, multi-merger system with a triple-nucleus and a $20 \mathrm{kpc}\left(\mathrm{H}_{0}=100 \mathrm{~km} \mathrm{~s}^{-1} \mathrm{Mpc}^{-1}\right)$ jet to the South. Also, a plume and faint tail extends $\sim 50 \mathrm{kpc}$ Northwest and there are more than 10 dwarf galaxies within $100 \mathrm{kpc}$ of Mrk 273 as determined from the POSS II R and J film copy. Therefore, the precursor of Mrk 273 is probably connected with a group of galaxies.

ROSAT PSPC observations show that the soft X-ray emission of Mrk 273 is extended up to $50 \mathrm{kpc}$. Moreover there exists an extended soft X-ray companion at a distance of about $30 \mathrm{kpc}$ that is certainly connected with Mrk 273 as revealed by our follow-up optical spectroscopic observation. The soft X-ray luminosity of the companion is $6.3 \times 10^{41} \mathrm{erg} \mathrm{s}^{-1}$ and its optical counterpart is an irregular dwarf galaxy as determined from its optical image and estimated apparent magnitude.

The soft X-ray luminosity for known irregular dwarfs is not as high as $10^{41} \mathrm{erg} \mathrm{s}^{-1}$ and the extended hot gaseous halo is not the established character of a Seyfert 2 galaxy. As Ponman et al. (1994, Nature, 369, 462) pointed out, an elliptical galaxy formed by the merger of a group will retain its halo. Therefore the hot gaseous halo and the soft X-ray companion of Mrk 273 very likely are a result of a multi-merger process and this may be evidence to support the merger origin for the intergroup or intercluster hot gas. 